Radiologe 2018 58 (Suppl 1):S7-S13 https://doi.org/10.1007/s00117-018-0409-1 Published online: 8 June 2018

(C) The Author(s) 2018 CrossMark

D. Leithner ${ }^{1,2} \cdot$ J. V. Horvat' $\cdot$ R. E. Ochoa-Albiztegui' $\cdot$ S. Thakur' $\cdot$ G. Wengert ${ }^{3}$ · E. A. Morris ${ }^{1} \cdot$ T. H. Helbich ${ }^{3} \cdot$ K. Pinker ${ }^{1,3}$

'Department of Radiology, Breast Imaging Service, Memorial Sloan Kettering Cancer Center, New York, USA

${ }^{2}$ Department of Diagnostic and Interventional Radiology, University Hospital Frankfurt, Frankfurt, Germany

${ }^{3}$ Department of Biomedical Imaging and Image-guided Therapy, Division of Molecular and Gender Imaging, Medical University Vienna, Vienna, Austria

\title{
Imaging and the completion of the omics paradigm in breast cancer
}

Within the field of oncology, "omics" strategies-genomics, transcriptomics, proteomics, metabolomics-have many potential applications and may greatly improve our understanding of the underlying processes of cancer development and progression. Omics strategies can play an important role in informing patient diagnosis, prognosis, and treatment $[1,2,40]$. In particular, they are naturally suited and highly promising for biomarker discovery as they allow for the rapid and simultaneous analysis of samples with rich biological information.

\section{Omics data in oncology}

In breast cancer (BC), the paradigm for omics strategies has always favored integrating multiple layers of omics data to achieve a complete portrait of BC. In the past decade, gene-expression profiling revolutionized $\mathrm{BC}$ classifications and replaced traditional categorizations based on immunohistochemistry with molecular subtypes (• Fig. 1; [10, 11]).

Four intrinsic molecular subtypes of $\mathrm{BC}$ have been revealed from extensive profiling at the DNA, microRNA, and protein levels by The Cancer Genome Atlas (TCGA) Network [5]: luminal A, luminal B, HER2(human epidermal growth factor receptor 2)-enriched, and triple negative (TN). Molecular BC subtypes are unevenly distributed in patients, are associated with different tumor phenotypes, and have a distinct prognosis, response to treatment, preferential metastatic organs, and recurrence or disease-free survival outcomes [18]. Since 2011, the St. Gallen International Expert Consensus panel has used the molecular subtype-based recommendations for systemic therapies for BC $[10$, 11].

Patients with the luminal A subtype have the most favorable prognosis, followed by patients with luminal $\mathrm{B}$, who have an intermediate prognosis. TN and HER2+ subtypes are associated with an unfavorable prognosis, but with the introduction of chemotherapy drugs such as trastuzumab and pertuzumab, the natural course of disease of TN and HER2+ has significantly improved [19]. Whereas luminal A cancers progress slowly over time with a greater chance of diseasefree survival for patients [20], luminal B, HER2+, and TN BCs tend to recur, with a peak incidence of recurrence within the first 5 years for luminal $B$ and the first 1-2 years for HER2+ and TN. Luminal cancers tend to metastasize to the bone while TN cancers metastasize to the viscera [33]. TN cancers are associated with a higher risk of regional relapse and the prognosis is dismal once the cancer spreads to regional lymph nodes, regardless of the number of nodes involved.

In the clinical and research settings, there is no readily available low-cost genetic testing to date and therefore molec- ular subtypes are commonly derived from invasive tissue sampling to guide therapy decisions. It should be noted that biopsies of small tumor regions are most likely not completely representative of the genetic, epigenetic, and/or phenotypic alterations of the entire tumor. In addition, although immunohistochemistry surrogates may provide clinical guidance, they have variable agreement with formal genetic testing (agreement rates have been reported to be between 41 and 100\%) and are less robust for predicting patient outcomes [16]. Therefore, there is a strong argument for an alternative, more accurate means of differentiating molecular BC subtypes and elucidating the underlying processes of BC development and progression, which poses a tremendous and unique opportunity for advanced medical imaging.

In this review, we discuss the pivotal role of radiogenomics in $\mathrm{BC}$ within the larger omics paradigm in $\mathrm{BC}$ oncology. We aim to give an overview of breast radiogenomics, its current role, future applications, and challenges.

\section{Radiogenomics in breast cancer}

Advances in medical imaging technologies, image analysis, and the development of high-throughput methods that can extract and correlate multiple imaging parameters with omics data have ushered in a new direction in medical re- 


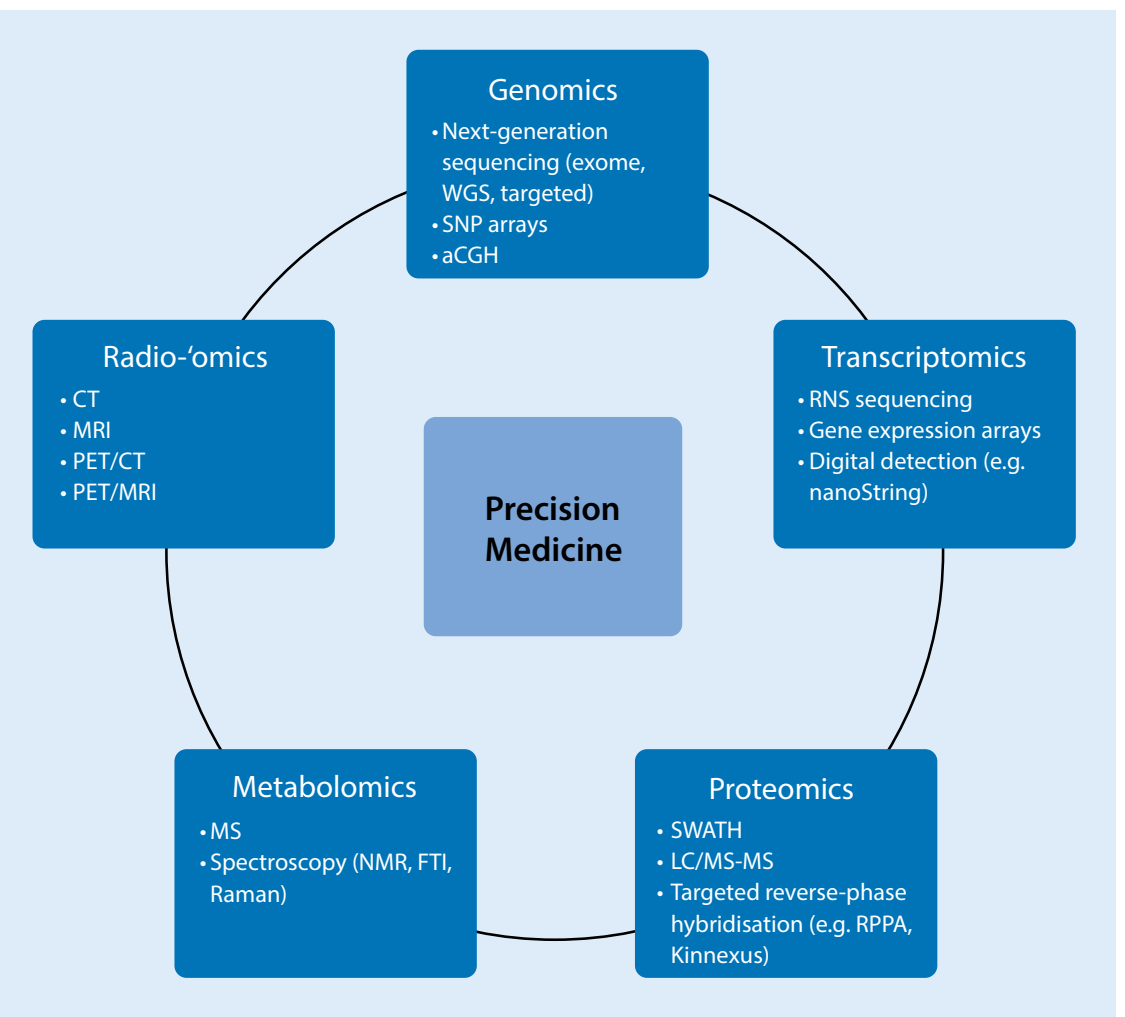

Fig. $1 \Delta$ Omics technologies used in the characterization of breast cancer for precision medicine. Molecular profiling and imaging technologies currently used for ome examination are shown. aCGH array comparative genomic hybridization, FTI Fourier-transform infrared, LC liquid chromatography, MS mass spectrometry, NMR nuclear magnetic resonance, RPPA reverse phase protein array, SNP single nucleotide polymorphism, SWATH serial window acquisition of theoretical spectra, WGS whole-genome sequencing. (Modified with permission from [22], this content is not part of the Open Access licence)

search. Radiogenomics is a relatively new omics strategy that correlates imaging characteristics (i.e., the imaging phenotype) with underlying gene expression patterns, gene mutations, and other genome-related characteristics $[4,6,9$, $17,26,27,32,44]$.

Radiogenomics is not synonymous with radiomics, which is defined as the conversion of medical images to higherdimensional, mineable data using computer classification algorithms and correlating these features with various data of interest such as patient characteristics, outcomes, and omics data for improved decision support $[9,25,27,32,38]$.

Radiogenomics not only represents the evolution in the radiology-pathology correlation from the anatomical-histological level to the molecular level, but it is a pivotal step in the omics paradigm in $\mathrm{BC}$ for fully characterizing the disease. With the use of modern analytical software tools, discoveries of new quan-

titative and qualitative imaging biomarkers offer hitherto unprecedented insights into the complex tumor biology and facilitate a deeper understanding of cancer development and progression. In a typical radiogenomics study, multiple qualitative and/or quantitative imaging features-i.e., shape, size, volume, signal intensity, or texture-are manually or (semi-)automatically extracted from an imaging dataset and are then correlated with omics data. This correlation provides useful bidirectional information: Imaging parameters can be used to predict cancer genotypes, and imaging phenotypes can be predicted from gene signatures $[4,8,26,32]$.

In 2012 the first article on radiogenomics in $\mathrm{BC}$ was published followed by a growing body of literature ever since [49]. So far, the rapidly evolving field of radiogenomics in $\mathrm{BC}$ has almost exclusively focused on magnetic resonance tool in breast imaging, with multiple indications such as preoperative staging, monitoring of neoadjuvant chemotherapy, and screening of high-risk patients. Dynamic contrast-enhanced MRI (DCEMRI) provides excellent morphologic information as well as limited functional information about abnormal vascularization as a tumor-specific feature. It is regarded as the most sensitive imaging modality for $\mathrm{BC}$ detection but has been criticized for its variable specificity. To add specificity and gain more functional information on $\mathrm{BC}$, diffusion-weighted imaging (DWI) has been developed and found to be an essential addition to DCE-MRI in multiple studies [37]. Today, multiparametric (mp) MRI including DCE-MRI and DWI has been successfully implemented into clinical routine. Additional parameters such as chemical exchange saturation transfer (CEST), blood oxygen level-dependent (BOLD), hyperpolarized (HP) MRI, and lipid MP spectroscopy (MRSI) are currently being developed and investigated. These newer applications in MRI promise to provide additional functional information and may open up further avenues for radiogenomics research.

\section{Feature extraction approaches} imaging (MRI). MRI is an established
For the purposes of radiogenomics analysis, imaging features can be extracted with human effort, semi-automatically or fully automatically using computer vision algorithms. Human feature extraction is based on image reading to provide specific variables such as lesion shape, margin, pattern, enhancement type, and kinetics; these features are defined by the American College of Radiology BI-RADS (Breast Imaging Reporting and Data System) MR lexicon. Human-extracted image variables are easily assessed but this process is time consuming and often limited by inter- and intra-observer variability, and thus semi- and fully automatic approaches should be preferred for feature extraction. While semi-automatic approaches still require human input in terms of tumor delineation or drawing the region of interest, fully-automatic computer-algorithm-extracted texture imaging features are of special interest for ra- 
Radiologe 2018· 58 (Suppl 1):S7-S13 https://doi.org/10.1007/s00117-018-0409-1

(c) The Author(s) 2018

\section{Leithner · J. V. Horvat · R. E. Ochoa-Albiztegui · S. Thakur · G. Wengert · E. A. Morris · T. H. Helbich · K. Pinker}

\section{Imaging and the completion of the omics paradigm in breast cancer}

\section{Abstract}

Within the field of oncology, "omics" strategies-genomics, transcriptomics, proteomics, metabolomics - have many potential applications and may significantly improve our understanding of the underlying processes of cancer development and progression. Omics strategies aim to develop meaningful imaging biomarkers for breast cancer $(\mathrm{BC})$ by rapid assessment of large datasets with different biological information. In BC the paradigm of omics technologies has always favored the integration of multiple layers of omics data to achieve a complete portrait of BC. Advances in medical imaging technologies, image analysis, and the development of high-throughput methods that can extract and correlate multiple imaging parameters with "omics" data have ushered in a new direction in medical research. Radiogenomics is a novel omics strategy that aims to correlate imaging characteristics (i.e., the imaging phenotype) with underlying gene expression patterns, gene mutations, and other genomerelated characteristics. Radiogenomics not only represents the evolution in the radiology-pathology correlation from the anatomical-histological level to the molecular level, but it is also a pivotal step in the omics paradigm in $\mathrm{BC}$ in order to fully characterize $B C$. Armed with modern analytical software tools, radiogenomics leads to new discoveries of quantitative and qualitative imaging biomarkers that offer hitherto unprecedented insights into the complex tumor biology and facilitate a deeper understanding of cancer development and progression. The field of radiogenomics in breast cancer is rapidly evolving, and results from previous studies are encouraging. It can be expected that radiogenomics will play an important role in the future and has the potential to revolutionize the diagnosis, treatment, and prognosis of $B C$ patients. This article aims to give an overview of breast radiogenomics, its current role, future applications, and challenges.

\section{Keywords}

Breast neoplasms · Magnetic resonance imaging · Diffusion-weighted imaging · Biomarkers - Gene expression · Molecular subtypes

\section{Bildgebung und die Vervollständigung des „Omics-Paradigmas“ bei Brustkrebs}

\section{Zusammenfassung}

Die "Omics-Strategien" - „genomics, transcriptomics, proteomics, metabolomics" haben viele potenzielle Anwendungsgebiete innerhalb der Onkologie und könnten das Verständnis der Krebsentstehung und des Fortschreitens der Erkrankung deutlich verbessern. Mit "Omics-Strategien" können große Datenmengen verschiedenster biologischer Informationen schnell ausgewertet werden, mit dem Ziel, sinnvolle Biomarker für Brustkrebs und hiermit ein integratives Porträt dieser Erkrankung zu entwickeln. Diese neue Richtung in der medizinischen Wissenschaft wurde durch verschiedenste Fortschritte in Bildgebungs- und Bildanalysemethoden sowie die Entwicklung von Techniken zur Extraktion und Korrelation verschiedenster Bildgebungsparameter mit "Omics-Daten" eingeläutet. Radiogenomics haben zum Ziel, Bildgebungscharakteristika (Phänotypen) mit Genexpressionsmustern, Genmutationen und weiteren genomassoziierten Eigenschaften zu korrelieren. Hiermit repräsentieren Radiogenomics die Evolution der Korrelation von Radiologie und Pathologie von der anatomisch-histologischen hin zur molekularen Ebene und stellen einen zentralen Schritt des „Omics-Paradigmas" bei Brustkrebs dar. In Verbindung mit modernen Softwareanalysetechniken können quantitative und qualitative Bildgebungsbiomarker bisher beispiellose Erkenntnisse über komplexe Tumorbiologie liefern und ein besseres Verständnis der Krebsentstehung und -progression bewirken. Radiogenomics stellen ein sich rasch entwickelndes Forschungsfeld dar, und Resultate aus ersten Studien sind vielversprechend. Es ist zu erwarten, dass Radiogenomics zukünftig eine wichtige Rolle spielen werden, da sie das Potenzial haben, Diagnose, Behandlung und Prognose von Brustkrebs zu beeinflussen. Im vorliegenden Artikel wird ein Überblick über Radiogenomics der Brust, ihre aktuelle Rolle, zukünftige Anwendungen und Herausforderungen gegeben.

\section{Schlüsselwörter}

Brustneoplasien - Magnetresonanztomographie - Diffusionsgewichtete Bildgebung . Biomarker · Genexpression · Molekulare Subtypen diogenomics, quantifying the morphology and three-dimensional (3D) structure of the lesion of interest on a voxelby-voxel basis.

Texture features are evaluated by texture analysis, which comprises four tasks with the aim of quantifying the morphology and internal structure of the tissue: feature extraction, texture discrimination, texture classification, and shape reconstruction [42]. In feature extraction, a numerical value is cal- culated based on statistical, structural, or model-based processing, e.g., with publicly available software such as the open source software MaZda (Technical University of Łodz, Institute of Electronics, Łodz, Poland; http://www.eletel.p. lodz.pl/programy/mazda/). In texture discrimination, images are segmented and regions with similar texture features are grouped together. These regions can be matched on predefined characteristics such as amount of fibroglandular tissue, benign tissue, or malignant breast lesions. The derived information can then be used to reconstruct $3 \mathrm{D}$ shapes and models and finally be correlated with genomic signatures or outcome variables.

In addition, data-mining algorithms can be used to extract dynamic variables such as enhancement kinetics, which allows for the assessment of neoangiogenesis as a tumor-specific feature. Kinetic features that are usually evaluated 


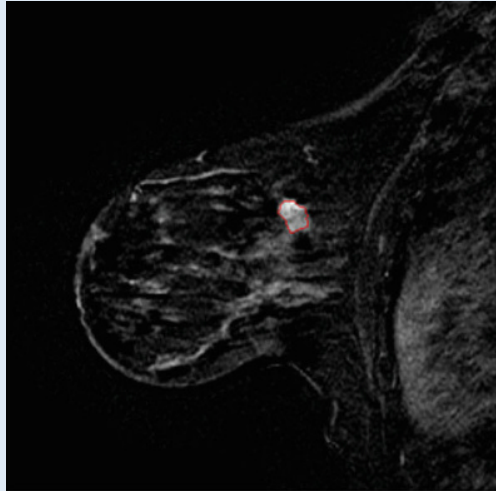

a

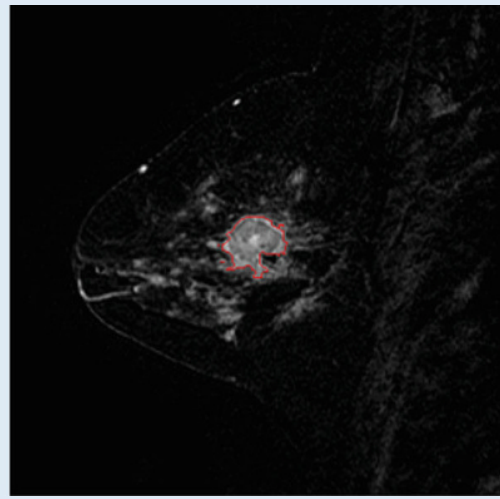

b

\begin{tabular}{|c|c|c|}
\hline & ER-Positive Case (a) & ER-Negative Case (b) \\
\hline Cancer Subtype & Luminal A & HER2-enriched \\
\hline $\begin{array}{c}\text { MRI CEIP Size } \\
\text { (Effective Diameter) } \\
\text { Range [7.8-54.0] }\end{array}$ & $12.9 \mathrm{~mm}$ & $23.8 \mathrm{~mm}$ \\
\hline $\begin{array}{c}\text { MRI CEIP Shape } \\
\text { (Irregularity) } \\
\text { Range [0.40-0.84] }\end{array}$ & 0.452 & 0.602 \\
\hline $\begin{array}{c}\text { MRI CEIP } \\
\text { Enhancement Texture } \\
\text { (Entropy) } \\
\text { Range [6.00-6.59] }\end{array}$ & 6.30 & 6.46 \\
\hline
\end{tabular}

C

Fig. $2 \Delta$ Example cases including segmentation outlines obtained from the computer segmentation method. a ER-positive example; $b$ ER-negative example; cCEIP values (and ranges) for size, irregularity, and enhancement texture of two example cases. CEIP computer-extracted image phenotypes, ER estrogen receptor, $H E R 2$ human epidermal growth factor receptor $2, M R /$ magnetic resonance imaging. (Reprinted with no modifications under a creative common license (https://creativecommons.org/ licenses/by/4.0/) from [29])

are the rate of enhancement on early postcontrast-enhanced sequences, peak enhancement, and late postcontrast-enhanced sequences.

Although computer vision algorithms are of special interest because they can facilitate the assessment of large data volumes, are not reader-dependent, and can provide information that is beyond human perception, they are not ready to be introduced into clinical routine, as research data are not yet fully reproducible owing to a lack of image protocol and data standardization.

\section{Radiogenomic approaches}

In exploratory radiogenomics studies, the extracted imaging features are tested against multiple different genomic characteristics, while metrics such as the false discovery rate are often used to detect meaningful prospective variables [12, 36, 39]. Hierarchical clustering is a method for evaluating similarities in large datasets and has been used famously in the original definition of the molecular subtypes of BC by Perou et al. [35]. In this approach, individual data points that show similarities are clustered until the relationship between all data points is established. The largest group at the top of the map is then used to define all groups within the dataset.

In hypothesis-driven radiogenomics studies, imaging characteristics are correlated with specific genetic signatures [26] with many potential benefits for BC diagnosis and therapy. As mentioned earlier, no low-cost genetic testing is available to date and the development of surrogates by means of radiogenomics with medical imaging is of great interest. In addition, radiogenomics might be used to develop imaging biomarkers to predict outcome parameters, such as therapy response or metastases [3].

\section{Current applications}

Thus far, MRI radiogenomics in the breast has mainly focused on DCE-MRI and the analyses of individual genomic signatures, BC molecular subtypes, or clinically used recurrence scores, with promising results.

\section{Individual genomic signatures}

In 2012, Yamamoto et al. conducted the first radiogenomics study in BC, demonstrating in ten $\mathrm{BC}$ patients that radiogenomics can be used to correlate gene expression patterns with imaging features in DCE-MRI [49]. In this groundbreaking study, the authors showed that 21 of 26 imaging characteristics were significantly associated with $71 \%$ of approximately 52,000 variably expressed genes. They found that 12 imaging characteristics were significantly correlated with BC genes, while 11 were significantly correlated with prognostic molecular characteristics. In a follow-up study by the same investigators using computer vision-extracted features and RNA sequencing, the enhancing rim fraction score was significantly associated with early metastasis [48].

Another group of authors, Zhu et al., investigated potential correlations of DCE-MRI features such as tumor size, shape, and morphology with genomic features such as transcriptional activities, protein expressions, and mutations for 91 breast carcinomas [51]. All selected DCE-MRI characteristics were associated with transcriptional activities of pathways, in particular tumor size, indicating that upregulated pathways are more common in large cancers. At the same time, associations between transcriptional activities and blurred tumor margins and irregular shape were found, indicating more aggressive malignancies.

\section{Molecular breast cancer subtypes}

As shown in recent efforts, radiogenomics has the potential to identify 


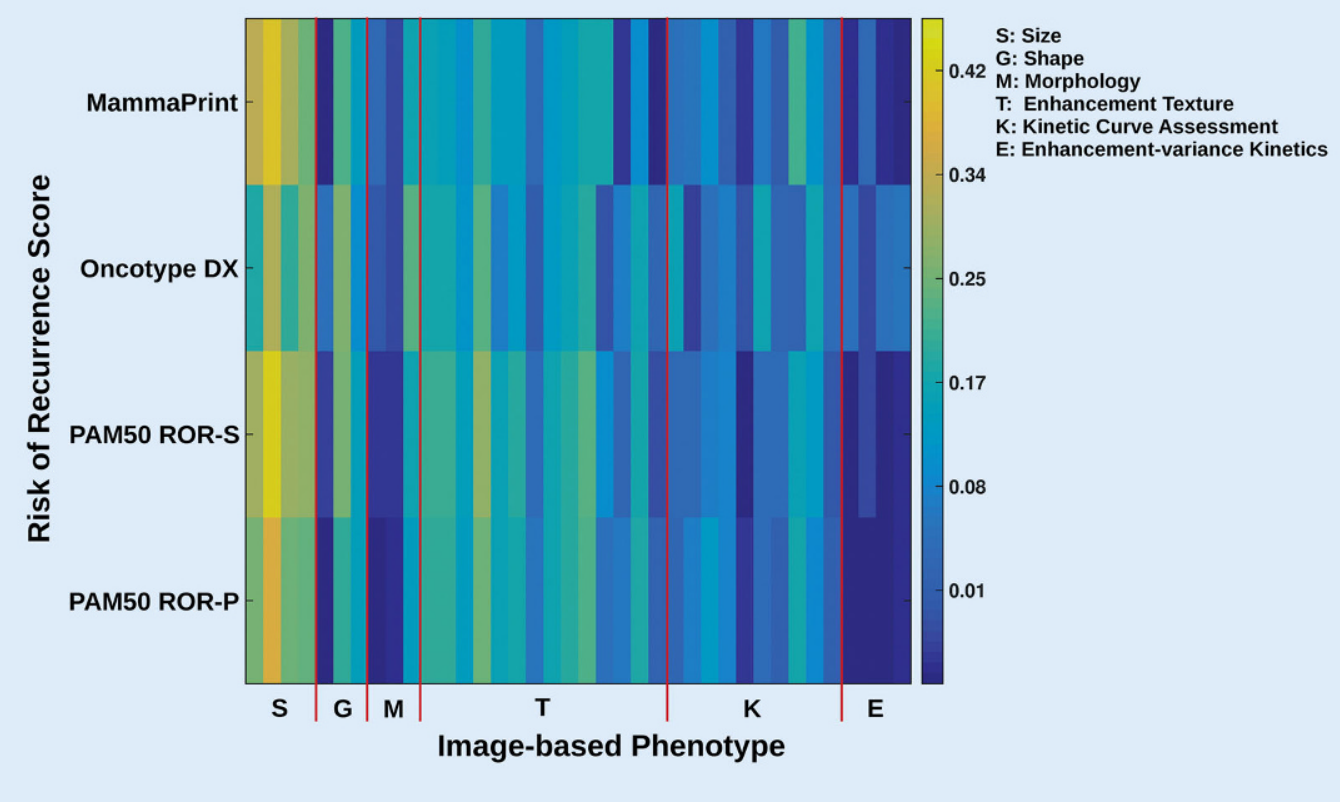

Fig. $3<$ Color map showing the correlation of MRI-based phenotypes with the recurrence predictor models of MammaPrint, OncotypeDX, PAM50 ROR-S, and PAM50 ROR-P. Here, yellow indicates higher correlation than blue. The different gene assays (recurrence predictor models) serve as "reference standard" in this study. $R O R$ - $P$ risk of relapse based on proliferation, $R O R-S$ risk of relapse based on subtype. (Reprinted with permission from [28], this content is not part of the Open Access licence) imaging biomarkers as reliable surrogates for genetic testing in the future. Grimm et al. found strong correlations between morphologic, kinetic, and textural imaging findings and luminal A and B subtypes [15]. While the classifier model by Waugh et al. had limited success, achieving an accuracy of $57.2 \%$ [46], Li et al. evaluated a classifier model utilizing tumor phenotypes to distinguish between molecular subtypes with promising results (• Fig. 2; [29]).

While the following are not radiogenomics studies, they show that imaging features are associated with molecular subtypes. In a recent study involving 278 cancer patients, Grimm et al. found significant correlations between DCEMRI BI-RADS descriptors and molecular subtypes [14]. Previous studies have also reported associations between DCEMRI enhancement kinetics and molecular BC subtypes. For instance, Elias et al. demonstrated that the luminal B subtype is associated with a higher internal enhancement of the tumor, while HER2-enriched cancers are more likely to show fast initial enhancement or wash-out kinetics [7]. HER2 subtypes have been described as being associated with a circumscribed margin, while TN subtypes are associated with rim enhancement and high $\mathrm{T} 2$ signal intensity $[13,45]$. In DWI, the highest apparent diffusion coefficient (ADC) values were found in HER2-enriched tumors, while luminal B/HER2-negative cancers showed the lowest ADC values [23, 31, 34], which might be due to the increased vascularization found in HER2-positive subtypes. These findings indicate that the assessment of functional tumor parameters with radiogenomics can be expected to contribute to our deeper understanding of $\mathrm{BC}$ biology.

\section{Recurrence scores}

Another clinically relevant application of radiogenomics is the correlation of imaging characteristics with prognostic genomic assays that provide scores for the risk of recurrence and are used to guide treatment decisions. Ashraf et al. demonstrated that DCE-MRI features indicative of greater tumor vascularization were associated with an increased risk of cancer recurrence [3]. Sutton et al. developed a model incorporating imaging and pathological information that showed a correlation with the OncotypeDx recurrence score [41]. In another study, Li et al. evaluated whether computerextracted imaging phenotypes could predict cancer recurrence using multigene assays, indicating that larger, more heterogeneous tumors have a higher risk for recurrence [28]. In this study, significant associations between $\mathrm{BC}$ MRI radiomics features and recurrence scores, especially MammaPrint, OncotypeDx, and PAM50/Prosigna, were found (• Fig. 3). In a very recent study, Woodard et al. evaluated the association of BI-RADS mammography and MRI features with $\mathrm{BC}$ recurrence in estrogen receptor (ER) positive patients using the OncotypeDx assay [47]. They found indistinct mass margins and fine linear branching calcifications to be significantly associated with a higher recurrence score, while breast density was inversely associated with the recurrence score (- Fig. 4 ). These studies illustrate that radiogenomics has the potential to identify multiple imaging biomarkers of $\mathrm{BC}$ recurrence risk, with larger studies needed to validate these preliminary findings.

\section{Challenges and future perspectives}

One of the main challenges of radiogenomics is the generation of big data, which must be stored, managed, and analyzed in a standardized, cost-effective way. Initiatives such as the Center for Advancing Translation Science of the National Institutes of Health (NIH, 2011) are being developed to address this problem. In addition, research in the field of radiogenomics is still limited by the interand intra-institutional data heterogeneity 


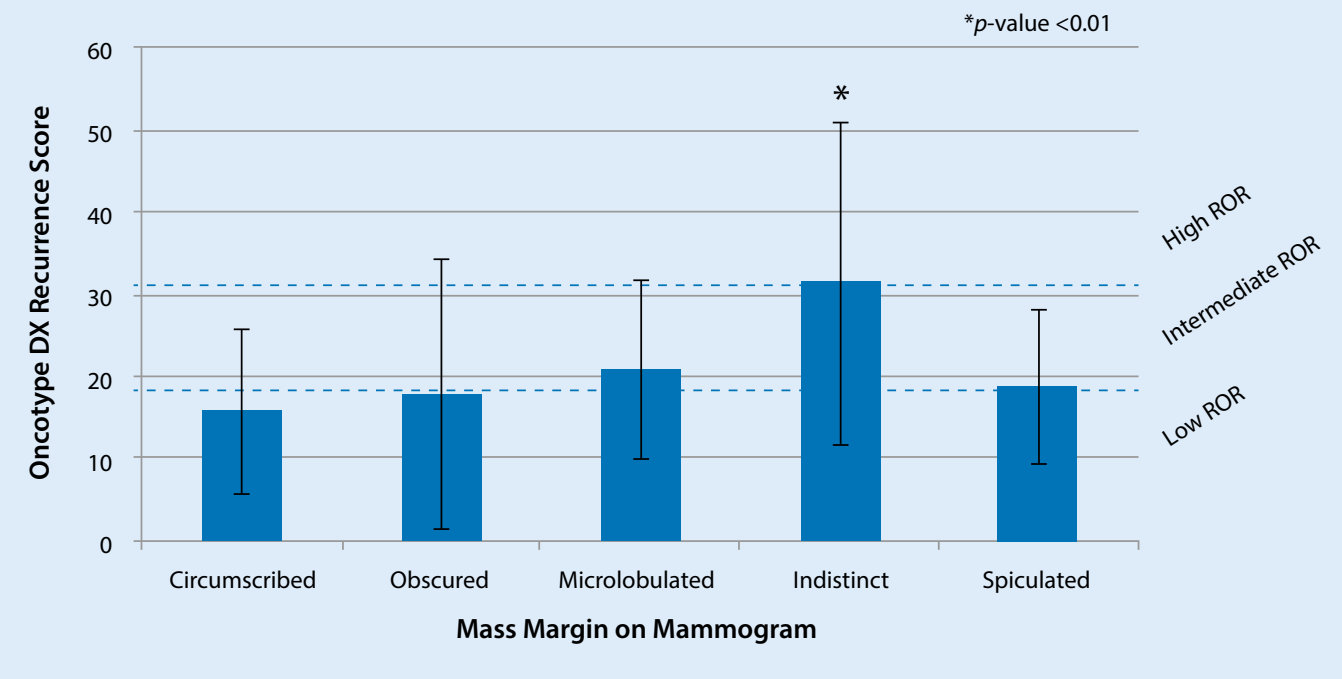

Fig. $4<$ Bar chart shows average ODxRS by using mammographic mass margins. Asterisk Indistinct mass margins versus all other mass margins; $p$ value adjusted for age at detection and personal history of invasive carcinoma or DCIS. ROR: low (score $<18$ ), intermediate (score 18-30), and high (score $\leq 31)$. DCIS ductal carcinoma in situ, ODxRS Oncotype DX test recurrence score, $R O R$ risk of recurrence. (Reprinted with permission from [47] this content is not part of the Open Access licence)

caused by different hardware, scan protocols, and postprocessing. Furthermore, genetic testing is challenging and costly, while the availability of genetic data is generally limited. The TCGA and Cancer Imaging Archive have been launched to store imaging and genetic data derived from different institutions. However, owing to these challenges, the conclusions that can be drawn from radiogenomic BC studies are limited by their mostly retrospective nature and small patient cohorts. To date, the evolving field of radiogenomics in breast imaging has almost exclusively focused on DCE-MRI, while most studies aimed to correlate genomic features with cancer subtypes and recurrence scores.

However, the field of imaging biomarkers development with MRI is rapidly growing. In DWI advanced techniques such as intravoxel incoherent motion, stretched exponential DWI, and DW kurtosis imaging are being investigated and hold promise for providing additional robust imaging biomarkers that can be incorporated in radiogenomic studies [30]. In addition, other MRI techniques that may be used for radiogenomic research include spectroscopy (proton, phosphorus, lipid), sodium imaging [50], CEST imaging [24], BOLD [21], and arterial spin labeling MRI [43]. Radiogenomics research in $\mathrm{BC}$ is still in its infancy. Larger prospective studies utilizing the full wealth of information that MRI can offer and considerable efforts in standardization and quality control are warranted, especially regarding outcome-related data, to meaningfully implement radiogenomics in the clinical setting.

\section{Practical conclusion}

- Radiogenomics examines the correlations of imaging phenotypes with characteristics derived from omics strategies-genomics, transcriptomics, proteomics, metabolomics.

- The integration of radiogenomics is a pivotal step toward completing the omics paradigm in BC.

- Thanks to the noninvasive nature and ubiquitous use of medical imaging in clinical routine, radiogenomics can elucidate disease processes by adding to our understanding of the disease etiology and helping to determine patient diagnosis, prognosis, and treatment.

- Exploration of additional functional imaging data in conjunction with omics technologies will open new avenues of multidimensional radiogenomic research.

- The implementation of radiogenomics in clinical BC care can further enhance the role of radiology. Additional efforts, rigorous standardization, and quality control are needed to validate already described radiogenomic correlations, discover new correlations, and define clinically relevant imaging biomarkers.

\section{Corresponding address}

\section{K. Pinker, MD, PhD}

Department of Radiology, Breast Imaging Service, Memorial Sloan Kettering Cancer Center

300 E 66th St, 7th Floor, 10065 New York, NY, USA

pinkerdk@mskcc.org

Funding. Open access funding provided by Medical University of Vienna.

\section{Compliance with ethical guidelines}

Conflict of interest. D. Leithner, J.V. Horvath, R.E. Ochoa-Albiztegui, S. Thakur, G. Wengert, E.A. Morris, T.H. Helbich and K. Pinker declare that they have no competing interests.

This article does not contain any studies with human participants or animals performed by any of the authors.

The supplement containing this article is not sponsored by industry.

Open Access. This article is distributed under the terms of the Creative Commons Attribution 4.0 International License (http://creativecommons.org/licenses/by/ 4.0/), which permits unrestricted use, distribution, and reproduction in any medium, provided you give appropriate credit to the original author(s) and the source, provide a link to the Creative Commons license, and indicate if changes were made. 


\section{References}

1. Abramovitz M, Leyland-Jones B (2006) A systems approach to clinical oncology: focus on breast cancer. Proteome Sci 4:5

2. Archer TC, Fertig EJ, Gosline SJ et al (2016) Systems approaches to cancer biology. Cancer Res 76:6774-6777

3. Ashraf AB, Daye D, Gavenonis S et al (2014) Identification of intrinsic imaging phenotypes for breast cancer tumors: preliminary associations with gene expression profiles. Radiology 272:374-384

4. Bai HX, Lee AM, Yang L et al (2016) Imaging genomics in cancer research: limitations and promises. Br J Radiol 89(1061):20151030. https:// doi.org/10.1259/bjr.20151030

5. CancerGenome Atlas Network (2012) Comprehensive molecular portraits of human breast tumours. Nature 490:61-70

6. Collins FS, Varmus H (2015) A new initiative on precision medicine. NEngl J Med 372:793-795

7. Elias SG, Adams A, Wisner DJ et al (2014) Imaging features of HER2 overexpression in breast cancer: a systematic review and meta-analysis. Cancer Epidemiol Biomarkers Prev 23:1464-1483

8. European Society of Radiology (ESR) (2010) White paper on imaging biomarkers. Insights Imaging $1: 42-45$

9. Gillies RJ, Kinahan PE, Hricak H (2016) Radiomics: images are more than pictures, they are data. Radiology 278:563-577

10. Goldhirsch A, Winer EP, Coates AS et al (2013) Personalizing the treatment of women with early breast cancer: highlights of the St Gallen International Expert Consensus on the Primary Therapy of Early Breast Cancer 2013. Ann Oncol 24:2206-2223

11. Goldhirsch A, Wood WC, Coates AS et al (2011) Strategiesforsubtypes-dealing with thediversity of breast cancer: highlights of the St. Gallen International Expert Consensus on the Primary Therapy of Early Breast Cancer 2011. Ann Oncol 22:1736-1747

12. Grimm LJ (2015) Breast MRI radiogenomics: current status and research implications. J Magn Reson Imaging 43(6):1269-1278. https://doi.org/ 10.1002/jmri.25116

13. Grimm LJ, Johnson KS, Marcom PKet al (2015) Can breast cancer molecular subtype help to select patients for preoperative MR imaging? Radiology 274:352-358

14. Grimm LJ, Zhang J, Baker JA et al (2017) Relationships between MRI Breast ImagingReporting and Data System (BI-RADS) lexicon descriptors and breast cancer molecular subtypes: internal enhancement is associated with luminal B subtype. Breast J 23(5):579-582. https://doi.org/ 10.1111/tbj.12799

15. Grimm LJ, Zhang J, Mazurowski MA (2015) Computational approach to radiogenomics of breast cancer: luminal A and luminal B molecular subtypes are associated with imaging features on routinebreastMRl extracted using computervision algorithms. J Magn Reson Imaging 42:902-907

16. Guiu S, Michiels S, Andre F et al (2012) Molecular subclasses of breast cancer: how do we define them? The IMPAKT 2012 Working Group Statement. Ann Oncol 23:2997-3006

17. Herold CJ, Lewin JS, Wibmer AGetal (2016) Imaging in the age of precision medicine: summary of the Proceedings of the 10th Biannual Symposium of the International Society for Strategic Studies in Radiology. Radiology 279:226-238
18. Huber KE, Carey LA, Wazer DE (2009) Breast cancer molecular subtypes in patients with locally advanced disease: impact on prognosis, patterns of recurrence, and response to therapy. Semin Radiat Oncol 19:204-210

19. IborraS, StickelerE(2016) HER2-orientated therapy in early and metastatic breast cancer. Breast Care (Basel) 11:392-397

20. Jatoi I, Anderson WF, Jeong JH et al (2011) Breast cancer adjuvant therapy: time to consider its timedependent effects. JClin Oncol 29:2301-2304

21. Jiang L, Weatherall PT, Mccoll RW et al (2013) Blood oxygenation level-dependent (BOLD) contrast magnetic resonance imaging (MRI) for prediction of breast cancer chemotherapy response: a pilot study. J Magn Reson Imaging 37:1083-1092

22. Kalita-De Croft P, Al-Ejeh F, Mccart Reed AE et al (2016) 'Omics' approaches in breast cancer research and clinical practice. Adv Anat Pathol 23:356-367

23. Kim EJ, Kim SH, Park GE et al (2015) Histogram analysis of apparent diffusion coefficient at $3.0 \mathrm{t}$ : correlation with prognostic factors and subtypes of invasive ductal carcinoma. J Magn Reson Imaging 42:1666-1678

24. Kogan F, Hariharan H, Reddy R (2013) Chemical Exchange Saturation Transfer (CEST) imaging: description of technique and potential clinical applications. Curr Radiol Rep 1:102-114

25. Kumar V, Gu Y, Basu S et al (2012) Radiomics: the process and the challenges. Magn Reson Imaging 30:1234-1248

26. Kuo MD, Jamshidi N (2014) Behind the numbers: decoding molecular phenotypes with radiogenomics - guiding principles and technical considerations. Radiology 270:320-325

27. Lambin P, Rios-VelazquezE, Leijenaar Ret al (2012) Radiomics: extracting more information from medical images using advanced feature analysis. Eur J Cancer 48:441-446

28. Li H, Zhu Y, Burnside ES et al (2016) MR imaging radiomics signatures for predicting the risk of breast cancer recurrence as given by research versions of mammaprint, oncotype DX, and PAM50 gene assays. Radiology 281:382-391

29. Li H, Zhu Y, Burnside ES et al (2016) Quantitative $\mathrm{MRI}$ radiomics in the prediction of molecular classifications of breast cancer subtypes in the TCGA/TCIA data set. NPJ Breast Cancer 2:16012

30. Mahajan A, Deshpande SS, Thakur MH (2017) Diffusion magnetic resonance imaging: a molecular imaging tool caught between hope, hype and the real world of "personalized oncology". World J Radiol 9:253-268

31. Martincich L, Deantoni V, Bertotto I et al (2012) Correlations between diffusion-weighted imaging and breast cancer biomarkers. Eur Radiol 22:1519-1528

32. Mazurowski MA (2015) Radiogenomics: what it is and why it is important. J Am Coll Radiol 12:862-866

33. Metzger-Filho O, Sun Z, Viale Getal (2013) Patterns of recurrence and outcome according to breast cancer subtypes in lymph node-negative disease: results from international breast cancer study group trials VIII and IX. JClin Oncol 31:3083-3090

34. Park SH, Choi HY, Hahn SY (2015) Correlations between apparent diffusion coefficient values of invasive ductal carcinoma and pathologic factors on diffusion-weighted MRI at 3.0 Tesla. J Magn Reson Imaging 41:175-182

35. Perou CM, Sorlie T, Eisen MB et al (2000) Molecular portraits of human breast tumours. Nature 406:747-752
36. Peterson $C B$, Bogomolov $M$, Benjamini $Y$ et al (2016) Many phenotypes without many false discoveries: error controlling strategies for multitrait association studies. Genet Epidemiol 40:45-56

37. Pinker K, BickelH, Helbich THetal (2013)Combined contrast-enhanced magnetic resonance and diffusion-weighted imaging reading adapted to the "Breast Imaging Reporting and Data System" for multiparametric 3-T imaging of breast lesions. Eur Radiol 23:1791-1802

38. Pinker K, Shitano F, Sala E et al (2018) Background, current role, and potential applications of radiogenomics. J Magn Reson Imaging 47:604-620

39. Reiner A, Yekutieli D, Benjamini Y (2003) Identifying differentiallyexpressedgenesusing falsediscovery rate controlling procedures. Bioinformatics 19:368-375

40. Sikkema AH, Den Dunnen WF, Diks SH et al (2012) Optimizing targeted cancer therapy: towards clinical application of systems biology approaches. Crit Rev Oncol Hematol 82:171-186

41. Sutton EJ, Dashevsky BZ, Oh JH et al (2016) Breast cancer molecular subtype classifier that incorporates MRI features. J Magn Reson Imaging 44:122-129

42. Szczypinski PM, Strzelecki M, Materka A et al (2009) MaZda-a software package for image texture analysis. Comput Methods Programs Biomed 94:66-76

43. Telischak NA, Detre JA, Zaharchuk G (2015) Arterial spin labeling MRI: clinical applications in the brain JMagn Reson Imaging 41:1165-1180

44. Thrall JH (2015) Moreton lecture: imaging in the age of precision medicine. J Am Coll Radiol 12:1106-1111

45. Uematsu T (2011) MR imaging of triple-negative breast cancer. Breast Cancer 18:161-164

46. Waugh SA, Purdie CA, Jordan LB et al (2016) Magnetic resonance imaging texture analysis classification of primary breast cancer. Eur Radiol 26:322-330

47. Woodard GA, RayKM, Joe BN etal (2018) Qualitative radiogenomics: association between oncotype DX test recurrence score and BI-RADS mammographic and breast MR imaging features. Radiology 286:60-70

48. Yamamoto S, Han W, Kim Y et al (2015) Breast cancer: radiogenomic biomarker reveals associations among dynamic contrast-enhanced MR imaging, long noncoding RNA, and metastasis. Radiology 275:384-392

49. Yamamoto S, Maki DD, Korn RL et al (2012) Radiogenomic analysis of breast cancer using MRI: a preliminary study to define the landscape. AJR Am J Roentgenol 199:654-663

50. Zaric O, Pinker K, Zbyn S et al (2016) Quantitative sodium MR imaging at $7 \mathrm{~T}$ : initial results and comparison with diffusion-weighted imaging in patients with breast tumors. Radiology 280:39-48

51. Zhu Y, LiH, GuoWetal(2015) Deciphering genomic underpinnings of quantitative MRI-based radiomic phenotypes of invasive breast carcinoma. Sci Rep 5:17787 